\title{
A Novel Hybrid PSO Identification Method Simultaneously Estimate Model Parameter and the Structure
}

\author{
Qibing Jin, Kewen Wang*, Jian Zhang and Liting Cao \\ Institute of Automation, Beijing University of Chemical Technology, Beijing, 100029, China
}

Received: 6 Aug. 2013, Revised: 8 Nov. 2013, Accepted: 9 Nov. 2013

Published online: 1 Jul. 2014

\begin{abstract}
Estimate model parameter and the structure simultaneously is a crucial and challenging problem in system identification. For solving the problem, a hybrid algorithm by integrating two-order oscillating particle swarm optimization with successive quadratic programming is proposed in this paper. The two-order oscillating particle swarm optimization is showed to converge rapidly to a near optimum solution, but the search process will become very slow around global optimum. On the contrary, successive quadratic programming is weak to escape local optimum but the ability of convergent speed around global optimum and the convergent accuracy is strong. In this case, the two-order oscillating particle swarm optimization is used to enhance global search ability and convergence speed of algorithm. When the change in fitness value is smaller than a threshold value, the searching process is switched to successive quadratic programming. In this way, this hybrid algorithm may find an optimum solution more accurately. To validate the performance of the proposed approach, it is evaluated on four optimal control problems. Results demonstrate the effectiveness and accuracy of the proposed algorithm.
\end{abstract}

Keywords: structural identification, parameter identification, combination optimization, hybrid particle swarm optimization, successive quadratic programming

\section{Introduction}

System identification is a challenging and complex optimization problem. It is a kind of theory to establish a mathematical model of the production process on the basis of input and output data. The kind of theory is gradual perfection and the practical application in many fields $[1,2]$. As time goes on, system identification theory has made rapid progress. But conventional identification methods have some drawbacks [3]. For instance using conventional methods directly for closed loop identification it may yield a large estimation bias, and even lead to unidentified. Later, some modern system identification methods such as identification based on neural-network, gene algorithm, fuzzy logic and wavelet network [4] are coming to handle these problems.

The particle swarm optimization (PSO) method has been introduced by Kennedy and Eberhart in 1995 [5]. It is a stochastic optimization method based on population which has merits of fast convergence, simple principle and easy implementation. Compare with other algorithms,
PSO algorithm has characters of simple computation and rapid convergence capability. For the reason of its high adaptability; PSO has many applications $[6,7,8,9,10]$. In fact, PSO has the problem of converging to undesired local solution or premature convergence. SQP is a nonlinear programming method that starts from a single searching point and finds a solution using the gradient information. Although this optimizing method is less time consuming than the population based search algorithms, it is highly dependent on the initial estimate of solution [11].

During the latest years, a multitude of implementations and modified versions have been proposed to correct the general PSO scheme presented above. Meimei Zhu et al. through chaotic initializing and detecting stagnation times of particles, and use global mutation strategy to the whole swarm, this method improved the convergent speed, obtained good results [12]. Lianguo Wang et al. purpose a hybrid algorithm by integrating particle swarm optimization (PSO) and

\footnotetext{
*Corresponding author e-mail: heaven996@ hotmail.com
} 
artificial fish swarm algorithm (AFSA) [13]. The algorithm divides the swarm into two sub-groups. One sub-group uses PSO algorithm and the other sub-group uses AFSA, and two algorithms share information with each other. Ximing Liang et al. analyze the contradiction of the global exploration and convergent speed of particle swarm optimization with dimension mutation operator, and an improved algorithm is proposed by modifying PSO with dimension mutation based on dynamical inertial weight vector [14]. In the proposed algorithm, the concept of dimension diversity is defined and inertial weight vector will be updated dynamically according to dimension diversity. The mutation operated on dimension whose dimension diversity is the worst. Yanmin Liu et al. through analyzing the relationship between swarm diversity and local optimum present an improved particle swarm optimizer based on dynamic neighbor topology (DPSO) [15] In DPSOthe neighbor of each particle is dynamically constructed at several iterationswhich increase the swarm diversity and improve the ability to escape from local optimum. Yuehong Sun et al. propose an improved PSO based on the diversity of particle symmetrical distribution (sdPSO) [16]. Through the research of the spatial distribution of particlesit can be found that the convergence probability to the global optimum solution is greatly improved with more symmetrical particle distribution surrounding the optimum solution of particlesA diversity population function is proposed and an adjustment algorithm for the diversity is introduced into the standard PSOThe spatial distribution of particles varies between asymmetry and symmetry repeatedly while the population diversity is adjusted continuallywhich make the improved algorithm search in a wider range. Shutao Li et al. propose a hybrid global optimization strategy combining PSO with a modified Broyden-Fletcher-Goldfarb-Shanno (BFGS) method [17]. The modified BFGS method is integrated into the context of the PSO to improve local search ability of the particles. In addition, in conjunction with the territory technique, a reposition technique to maintain the diversity of particles is proposed to the hybrid strategy is that it can effectively find multiple local solutions or global solutions to the multimodal functions in a box-constrained space. Based on these local solutions, a reconstruction technique can be adopted to further estimate better solutions. Jiuzhong Zhang et al. propose a multi-swarm self-adaptive and cooperative particle swarm optimization (MSCPSO) based on four sub-swarms [18]. In this method several strategies are employed to avoid falling into local optimum, improve the diversity and achieve better solution. Particles in each sub-swarms share the only global historical best optimum to enhance the cooperative capability. Besides, the inertia weight of a particle in each sub-swarm is modified, which is subject to the fitness information of all particles, and the adaptive strategy is employed to control the influence of the historical information to create more potential search ability. To effectively keep the balance between the global exploration and the local exploitation.

Motivated by the aforementioned researches, the goal of this paper is to present a novel algorithm based on two-order oscillating with random inertia weight particle swarm optimization (TPSO) and successive quadratic programming (SQP) for estimating model parameter and the structure simultaneously. The feasibility of this algorithm is demonstrated through identifying the parameters of single variable system, multi variable system, Hammerstein model and Wiener model. The performance of the proposed method is compared with the standard PSO algorithm and the dynamical inertia weight of PSO algorithm in terms of parameter accuracy. It is illustrated in simulations that the proposed method is more successful than the BPSO and WPSO.

\section{Identification problems description}

There are many factors to affect system outputs, especially in unknown system. System identification in this paper we discuss is the best fitting model according to the combination of sample data and can determine the parameter for the model.

Considering a class of multi-input and single output static systems, $y$ is system output variable, there are $m$ input variables, and they may affect the system. Sample data obtain from the system can be described as follow

$$
y_{i}, x_{1 i}, x_{2 i}, \cdots, x_{j i}, \cdots, x_{m i}
$$

where $x_{j i}$ is $i$-th group and $j$-th sample data in specimen, $y_{i}$ is output value of $i$-th group, $j=1,2, \cdots, m$, $i=1,2, \cdots, n$. Assume sample data model is composed by various kinds of sub-models, and sub-models are composed by meta-models.

Definition 2.1. Assume a single variable $x_{i}$ effect system output by $f\left(x_{i}\right)$ form, we call $f\left(x_{i}\right)$ is a single variable meta-model. For instance meta-model $f\left(x_{1}\right)=b x_{1}$ describes a linear function, output variable $y$ and $x_{1}$ are linear relation, where $b$ is meta-model parameter. Assume the number of meta-models is $N_{1}$, consider the input variables may be influenced output variables by many forms. Sample data models can be described as below.

$$
y=P_{0}+\sum_{k=1}^{N_{1}} \sum_{i=1}^{m} f_{k}\left(x_{i}\right)
$$

where $P_{0}$ is constant.

Definition 2.2. Assume variables $x_{i}$ and $x_{j}$ influenced system output by form $f\left(x_{i}, x_{j}\right), x_{i} \neq x_{j}$ and $f\left(x_{i}, x_{j}\right)$ cannot be decomposed to $f\left(x_{i}\right)+f\left(x_{j}\right)$. We call $f\left(x_{i}, x_{j}\right)$ as double variables meta-model. For instance, $f\left(x_{i}, x_{j}\right)=a x_{1}^{b} x_{2}^{c}$ is a production function meta-model, output variables $y, x_{i}$ and $x_{j}$ had production function relation, where $a, b$ and $c$ are meta-model parameters. 
Assume the number of meta-models is $N_{2}$, consider the input variables may be influenced output variables by various types of model forms. We described sample data models as below.

$$
y=P_{0}+\sum_{k=1}^{N_{1}} \sum_{i=1}^{m} f_{k}\left(x_{i}\right)+\sum_{k=1}^{N_{2}} \sum_{i=1}^{m} \sum_{j=1}^{n} f_{k}\left(x_{i}, x_{j}\right)
$$

It can be seen that the number of variables, meta-models and independent variables of meta-model is increased, sub-model combination numbers is increased exponentially. Once the meta-model is confirmed, the number of sub-model in sample data model also can be confirmed. In conclusion, the general form of the sample data model can be described as below:

$$
y=P_{0}+\sum_{k=1}^{N_{1}} M_{k}\left(x, p_{k 1}, p_{k 2}, \cdots, p_{k m_{k}}\right)
$$

where $\sum_{k=1}^{N_{1}} M_{k}\left(x, p_{k 1}, p_{k 2}, \cdots, p_{k m_{k}}\right)$ is the $k$-th sub-model constructed by meta-models and variables, $x$ can both describe single variable and multi variable, $m_{k}$ are parameter numbers in this model, $k=1,2, \cdots, N, P_{k m_{j}}$ is the $j$-th parameter in $M_{k}\left(x, p_{k 1}, p_{k 2}, \cdots, p_{k m_{k}}\right)$, $j=1,2, \cdots, m_{k}, P_{0}$ is constant, $N$ is the number of sub-models.

In nonlinear system, Hammerstein model and Wiener model are two kinds of typical nonlinear system. Hammerstein model consists of two parts: linear part and nonlinear part. The construction of Hammerstein model is shown in figure 1.

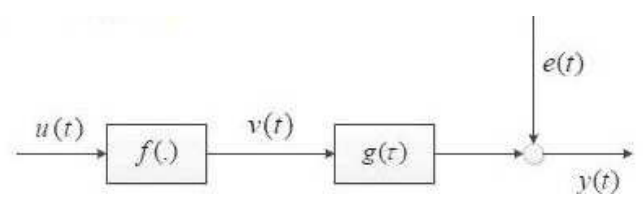

Fig. 1: Hammerstein Model

Where $f(\cdot)$ is nonlinear part, $g(\tau)$ is linear part. The nonlinear part can be described by the formula 5 :

$$
f[u(t)]=1+a_{1} u(t)+a_{2} u^{2}(t)+\cdots+a_{q} u^{q}(t)
$$

where $u(t)$ stands for input of system, $v(t)$ indicates the output of nonlinear part, and also represents the input of linear sub-system.

$$
v(t)=u(t) \cdot f[u(t)]
$$

Linear sub-model is described by impulse response function:

$$
y(t)=\sum_{i=1}^{n} g_{i} v(t-i)+e(t)
$$

where $n$ is the adjust time for linear sub-model. When $i>$ $n, g_{i}=0$. Assume the average of noise $e(t)$ is 0 . It means $E\{e(t)\}=0$.

Nonlinear system Wiener model is combined with linear sub-model and nonlinear sub-model shown in figure 2 .

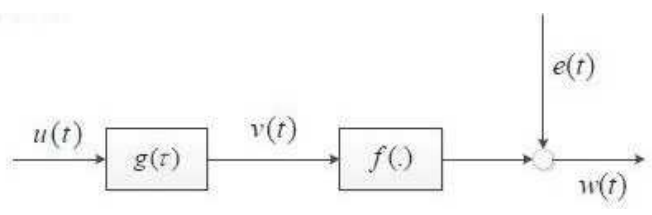

Fig. 2: Wiener Model

Nonlinear part we can use formula 8 to describe:

$$
f[y(t)]=1+a_{1} y(t)+a_{2} y^{2}(t)+\cdots+a_{q} y^{q}(t)
$$

Assume the average of system noise $e(t)$ is 0 and is a smooth time sequence. For easily to compute, we set $e(t) \equiv 0$, the system output is:

$$
w(t)=y(t) \cdot f[y(t)]
$$

We use discrete impulse response function to describe the linear part of Wiener model.

$$
y(t)=\sum_{\tau=0}^{n} g_{\tau} u(t-\tau)
$$

\section{Preliminaries: the classic optimization algorithms - TPSO and SQP}

\subsection{Two-order oscillating with random inertia weight particle swarm optimization (TPSO)}

In standard PSO algorithm, particle speed is only the function of current position. However in second order particle swarm optimization algorithm particle speed is related to the position alteration.

The updating forum is as follows:

$$
\begin{aligned}
v_{i, j}(t+1)= & w_{i, j}(t)+c_{1} r_{1}\left[p_{i, j}-2 x_{i, j}(t)+x_{i, j}(t-1)\right] \\
& +c_{2} r_{2}\left[p_{g, j}-2 x_{i, j}(t)+x_{i, j}(t-1)\right]
\end{aligned}
$$

Second order particle swarm optimization algorithm is asymptotic convergence. In order to enhance the diversity of swarm, an oscillation segment is introduced to improve global convergence ability of algorithm. The updating forum is as follows:

$$
\begin{aligned}
& v_{i, j}(t+1)=w v_{i, j}(t)+c_{1} r_{1}\left[p_{i, j}-\left(1+\xi_{i}\right) x_{i, j}(t)\right. \\
& \left.+\xi_{1} x_{i, j}(t-1)\right]+c_{2} r_{2}\left[p_{g, j}-\left(1+\xi_{2}\right) x_{i, j}(t)+\xi_{2} x_{i, j}(t-1)\right]
\end{aligned}
$$




$$
x_{i, j}(t+1)=x_{i, j}(t)+v_{i, j}(t+1)
$$

where $\xi_{1}$ and $\xi_{2}$ are random numbers. In the global search algorithm, we usually expect that the algorithm has excellent global search capability in the early of optimization process to avoid the local search trap or premature convergence, and later stage of optimization process we expect the algorithm has powerful local search ability and fast convergence speed. Based on these we take $\xi_{1}<\frac{2 \sqrt{c_{1} r_{1}}-1}{c_{1} r_{1}}$ and $\xi_{2}<\frac{2 \sqrt{c_{2} r_{2}}-1}{c_{2} r_{2}}$ in the early stage of algorithm and later stage of optimization process we take $\xi_{1} \geq \frac{2 \sqrt{c_{1} r_{1}}-1}{c_{1} r_{1}}$ and $\xi_{2} \geq \frac{2 \sqrt{c_{2} r_{2}}-1}{c_{2} r_{2}}$ can make the algorithm asymptotic convergence.

Consider the complexity of nonlinear system. The algorithm may not have accuracy estimation result. Further we improve the inertial weight. A random inertial weight is introduced to two-order oscillating particle swarm optimization. We set inertia $w$ is a random number which obey certain random distribution. This can overcome $w$ linear decrease shortage from two aspects.

First of all, if at the early of evolution process closed to the best point, random $w$ may generate small value to accelerate the convergence speed. In addition, if the best point can't be found at the early stage, algorithm will not converge at the best point with $w$ linear decrease.

The updating forum is as follows:

$$
\left\{\begin{array}{l}
w=\mu+\sigma \times N(0,1) \\
\mu=\mu_{\text {min }}+\left(\mu_{\max }-\mu_{\min }\right) \times \operatorname{rand}(0,1)
\end{array}\right.
$$

where $N(0,1)$ stands for standard normal distribution random number, and $\operatorname{rand}(0,1)$ indicates random number between 0 and 1 .

\subsection{Successive quadratic programming (SQP)}

The method resembles closely to Newton's method for constrained optimization just as is done for unconstrained optimization. SQP is based on iterative formulation and on the solution of quadratic programming sub-problems. The sub-problem is obtained by linearizing the constraints and approximating the Lagrangian function quadratically:

$$
L(x, \lambda)=J(x)+\sum_{i=1}^{m} \lambda_{i} \psi_{i}(x)
$$

At each iteration, an approximation of the Hessian of the Lagrangian function $H_{k}$ is made.

The process starts from given iteration $x_{k}$, then, the following quadratic programming (QP) sub-problem is formed to solve:

$$
\begin{gathered}
\min \frac{1}{2} d^{T} H_{k} d+\nabla\left(f x_{k}\right)^{T} d \\
\nabla \psi_{i}\left(x_{k}\right)^{T} d+\psi_{i}\left(x_{k}\right)=0, i=1, \cdots, m_{e}
\end{gathered}
$$

$$
\nabla \psi_{i}\left(x_{k}\right)^{T} d+\psi_{i}\left(x_{k}\right) \geq 0, i=m_{e}, \cdots, m \quad d \in R^{n}
$$

This sub-problem is a quadratic programming (QP) sub-problem whose solution is used to form a search direction for a line search procedure. In other words, the solution is used to form the next iterate:

$$
x_{k+1}=x_{k}+\alpha_{k} d_{k}
$$

The step length parameter is determined by an appropriate line search procedure so that a sufficient decrease in a merit function is obtained. The method is vastly used in optimization problems, but it is also known that it depends on the initial estimate.

\section{A novel algorithm TPSO-SQP and its application in the nonlinear identification}

TPSO-SQP method proposed by this paper divides system identification into system structure identification and parameters identification. Structure identification is a kind of selection sub-model problem. Choose a sub-model of best fitting sample data is a kind of combinatorial optimization problem. The combinations increase with the number of mathematical models. The optimization calculation workload also increases greatly. PSO algorithm is a global algorithm, which has a strong ability to find global optimistic result. However, it has a disadvantage that the search around global optimum is very slow. The SQP algorithm, on the contrary, has strong ability to find local optimistic result for nonlinear system identification problem, but its ability to find the global optimistic result is weak. By combining the TPSO with SQP, a new algorithm referred to as TPSO-SQP hybrid algorithm is formulated in this paper. Similar to the PSO algorithm, the TPSO-SQP algorithm's searching process is also started from initializing a group of random particles. First, TPSO algorithm is run to search the global best position in the solution space. Then SQP algorithm is used to search around the global optimum. In this way, this hybrid algorithm may find an optimum more quickly and accurately. TPSO-SQP algorithm provides a novel method to estimate model parameter and the structure simultaneously. The procedure for this TPSO-SQP algorithm can be summarized as follows:

Step 1: Initialize the positions and velocities of a group of particles randomly.

Step 2: Evaluate each initialized particle's fitness value

Step 3: If the maximal iterative iterations are arrived, go to Step 7, else, go to Step 4.

Step 4: The best particle of the current particles is stored. If the change between the current best particle fitness value and its previous one is smaller than a predefined value, go to step 7, else continue. 
Step 5: The positions and velocities of all the particles are updated according to Eq. (12) and Eq. (13), and then a group of new particles is generated.

Step 6: Update the inertia weight for each particle according to Eq. (14) and go to step 2.

Step 7: Use SQP algorithm to search around global best, which is found by TPSO to find finer solutions. In this case, the best solution obtained by TPSO is considered as the initial guess for SQP algorithm.

\section{Simulation Research}

\subsection{Meta-Models selection}

Sample data model is composed of sub-models, and each sub-model is composed of meta-model and independent variable, so the selection of meta-models playing a decisive role in system identification. Meta-model selection should obey the following principles: 1) Universality, means the model often appears in related fields. 2) Representativeness, means each model have different characteristics. 3) Encompassment, means through the combination of meta-model, can contain relations and rules as many as possible.

We select these typical meta-models in this paper,: linear model $y=b x$, exponential function model $y=a e^{b x}$, negative exponential utility function $y=a e^{b / x}$, power function model $y=a x^{b}$, logarithmic function $y=a \ln (b+x)$, hyperbolic function model $y=a /(b+x)$, Logistic model $y=1 /\left(a+b c^{x}\right)$, Gompertz model $y=k a^{b x}, S$ model $y=1 /\left(a+b e^{-c x}\right)$, periodic function model $y=a \sin (b+c x)$.

\subsection{Single variable system model}

Transfer function of single variable system model is

$$
y=2+1.5 e^{-1.25 x}-1.5 \sin (1.8+1.5 x)
$$

According to Eq. (20) generates 30 groups sample model, and we don't know the model structure. In this condition, we use meta-models above to identify model structure. Set the parameters of BPSO algorithm as follow: particle numbers $N=20$, inertia weight $w=1$, accelerate factors $c_{1}=2, c_{2}=1.65, w_{\max }=2$, the $\max$ iteration number is 1000 . In WPSO algorithm set inertia weight as follow: $w_{\max }=0.9, w_{\min }=0.4$. Other parameters are the same as BPSO algorithm.

After simulation, we choose the best result as follow: BPSO algorithm:

$$
\begin{aligned}
y= & 2.000+2.000 e^{-2.000 x} \\
& -1.4991 \sin (2.000+1.4903 x)
\end{aligned}
$$

WPSO algorithm:

$$
\begin{aligned}
y= & 2.000+1.324 e^{-2.000 x} \\
& -1.5069 \sin (1.8201+1.4990 x)
\end{aligned}
$$

TPSO-SQP algorithm:

$$
\begin{aligned}
y= & 2.000+1.4998 e^{-1.2500 x} \\
& -1.5000 \sin (1.8000+1.5000 x)
\end{aligned}
$$

Identification parameters of each algorithm are shown in table 1 , mean squares of each algorithm are shown in table 2.

Table 1: Identification parameters of each algorithm

\begin{tabular}{ccccccc}
\hline Truth Value & 2 & 1.5 & -1.25 & -1.5 & 1.8 & 1.5 \\
\hline BPSO & 2.000 & 2.000 & -2.000 & -1.4991 & 2.000 & 1.4903 \\
WPSO & 2.000 & 1.324 & -2.000 & -1.5069 & 1.801 & 1.4990 \\
TPSO-SQP & 2.000 & 1.4998 & -1.2500 & -1.5000 & 1.8000 & 1.5000 \\
\hline
\end{tabular}

Table 2: Mean squares of each algorithm

\begin{tabular}{ccc}
\hline & maximum deviation & mean-square deviation \\
\hline BPSO & 0.7500 & 0.0295 \\
WPSO & 0.7500 & 0.0017 \\
TPSO-SQP & 0.0002 & $2.0954 \mathrm{e}-009$ \\
\hline
\end{tabular}

The comparison of system output is shown in figure 3 . We can see that the results of the presented method are approximate to the true process model.

\subsection{Multi-variable system model}

Transfer function of single variable system model is

$$
y=1+x_{1}-1.5 x_{2}+1.6 e^{-x_{3}}+2 \sin \left(1+x_{4}\right)
$$

According to Eq. (21) generates 30 groups sample model, and we don't know the model structure. In this condition, we use meta-models above to identify model structure. Set the parameters of BPSO algorithm as follow: particle numbers $N=20$, inertia weight $w=1$, accelerate factors $c_{1}=2, c_{2}=1.65, w_{\max }=2$, the $\max$ iteration number is 1200 . In WPSO algorithm set inertia weight as follow: $w_{\max }=0.9, w_{\min }=0.4$. Other parameters are the same as BPSO algorithm.

After simulation, we choose the best result as follow: BPSO algorithm is

$$
\begin{aligned}
y= & 0.2548+1.4775 x_{1}-1.9802 x_{2}+1.9991 e^{-1.3560 x_{3}} \\
& +2.1390 \sin \left(1.9882+1.0138 x_{4}\right)
\end{aligned}
$$




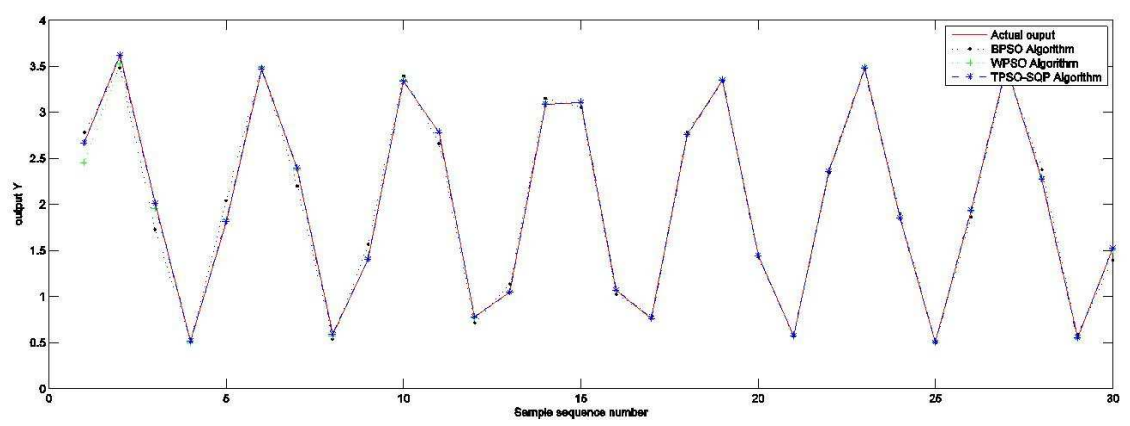

Fig. 3: The results of some methods

Table 3: Identification parameters of each algorithm

\begin{tabular}{ccccccccc}
\hline Truth Value & 1 & 1 & -1.5 & 1.6 & -1 & 2 & 1 & 1 \\
\hline BPSO & 0.2548 & 1.4775 & -1.9802 & 1.9991 & -1.3560 & 2.1390 & 1.9882 & 1.0138 \\
WPSO & 0.3793 & 1.0683 & -1.5760 & 1.2469 & -0.8776 & 2.0376 & 0.9791 & 1.0013 \\
TPSO-SQP & 0.9970 & 0.9956 & -1.5042 & 1.5896 & -0.9734 & 2.0027 & 0.9988 & 1.0001 \\
\hline
\end{tabular}

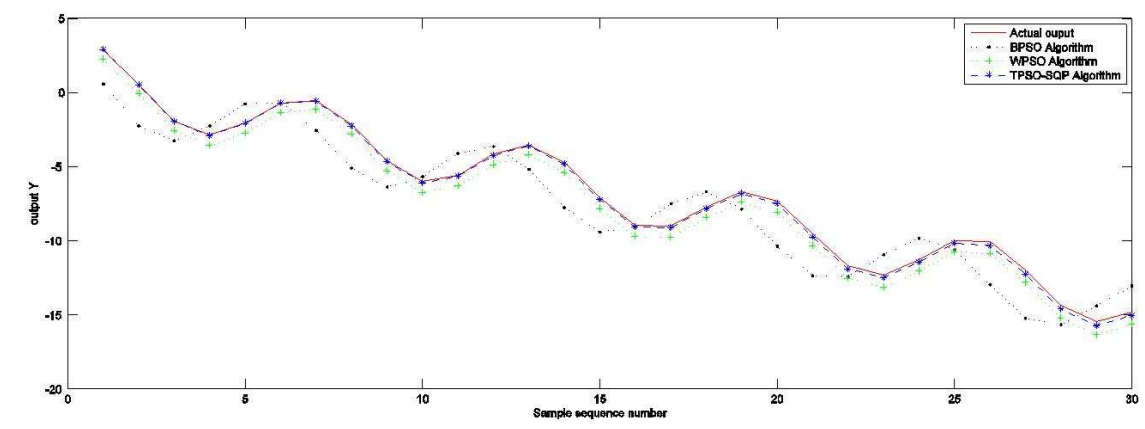

Fig. 4: The results of some methods

WPSO algorithm is

$$
\begin{aligned}
y= & 0.3793+1.0683 x_{1}-1.5760 x_{2}+1.2469 e^{-0.8776 x_{3}} \\
& +2.0376 \sin \left(0.9791+1.0013 x_{4}\right)
\end{aligned}
$$

TPSO-SQP algorithm is

$$
\begin{aligned}
y= & 0.9970+0.9956 x_{1}-1.5042 x_{2}+1.5896 e^{-0.9734 x_{3}} \\
& +2.0027 \sin \left(0.9988+1.0001 x_{4}\right)
\end{aligned}
$$

Identification parameters of each algorithm are shown in table 3 , mean squares of each algorithm are shown in table 4 .

The comparison of system output is shown in figure 3 . We can see that the results of the presented method are approximate to the true process model.

From the contrasts above we could see, the hybrid algorithm combined particle swarm optimization algorithm and sequential quadratic programming
Table 4: Mean squares of each algorithm

\begin{tabular}{ccc}
\hline & maximum deviation & mean-square deviation \\
\hline BPSO & 0.9882 & 0.7285 \\
WPSO & 0.6207 & 0.0123 \\
TPSO-SQP & 0.0266 & $8.6036 \mathrm{e}-005$ \\
\hline
\end{tabular}

algorithm identify system output curve is more close to the real value, precision obviously improved.

\subsection{Identification of Hammerstein model}

Consider the following Hammerstein model

$$
\left\{\begin{array}{l}
v(t)=u(t)+u^{2}(t) \\
y(t)=1.5 y(t-1)-0.7 y(t-2)+v(t-1) \\
-0.8 v(t-2)+e(t)
\end{array}\right.
$$


Table 5: Identification parameters of each algorithm

\begin{tabular}{ccccccc}
\hline Truth Value & 1 & 1 & 1.5 & -0.7 & 1 & -0.8 \\
\hline BPSO & 1.1149 & 0.9378 & 1.5443 & -0.6618 & 0.7447 & -0.6038 \\
WPSO & 1.0822 & 1.1662 & 1.4811 & -0.6973 & 0.9828 & -0.6726 \\
TPSO-SQP & 0.9973 & 0.9567 & 1.5004 & -0.7028 & 1.0538 & -0.8463 \\
\hline
\end{tabular}

Table 6: Mean squares of each algorithm

\begin{tabular}{ccc}
\hline & maximum deviation & mean-square deviation \\
\hline BPSO & 0.2553 & 0.9730 \\
WPSO & 0.6726 & 1.1175 \\
TPSO-SQP & 0.0538 & 1.0022 \\
\hline
\end{tabular}

Table 7: Identification parameters of each algorithm

\begin{tabular}{ccccccc}
\hline Truth Value & 1 & 1 & 1.5 & -0.7 & 1 & -0.8 \\
\hline BPSO & 1.6058 & 1.5055 & 1.0484 & -0.3257 & 0.7082 & -0.1840 \\
WPSO & 1.4337 & 1.3927 & 1.8962 & -0.4973 & 0.6967 & -0.3512 \\
TPSO-SQP & 0.7513 & 0.7298 & 1.5708 & -0.7712 & 0.8712 & -0.5749 \\
\hline
\end{tabular}

Table 8: Mean squares of each algorithm

\begin{tabular}{ccc}
\hline & maximum deviation & mean-square deviation \\
\hline BPSO & 0.9267 & 1.2299 \\
WPSO & 0.4962 & 0.9719 \\
TPSO-SQP & 0.2702 & 0.9129 \\
\hline
\end{tabular}

A Gaussian white noise is added to the output to test the robustness. We choose noise signal $e(t)$ which variance is 0.1 and mean value is 0 , pumping signal $u(t)$ is:

$$
u(t)=\frac{1}{20}\left[\sin \left(\frac{2 \pi}{250} t\right)+\sin \left(\frac{2 \pi}{25} t\right)\right]
$$

The purpose of system identification is system output we obtained as close as the known system output, the closer is the better. We take principle function as follow:

$$
f=\sum_{t}\left[y(t)-y_{0}(t)\right]^{2}
$$

In noiseless condition, after simulation, choose the best result as follows:

BPSO algorithm

$$
\left\{\begin{array}{l}
v(t)=1.1149 \cdot u(t)+0.9378 \cdot u^{2}(t) \\
y(t)=1.7443 \cdot y(t-1)-0.6618 \cdot y(t-2) \\
+0.7447 \cdot v(t-1)-0.6038 \cdot v(t-2)+e(t)
\end{array}\right.
$$

WPSO algorithm

$$
\left\{\begin{array}{l}
v(t)=1.0822 \cdot u(t)+1.1662 \cdot u^{2}(t) \\
y(t)=1.4811 \cdot y(t-1)-0.2973 \cdot y(t-2) \\
+0.5828 \cdot v(t-1)-1.4726 \cdot v(t-2)+e(t)
\end{array}\right.
$$

TPSO-SQP algorithm

$$
\left\{\begin{array}{l}
v(t)=0.9973 \cdot u(t)+0.9567 \cdot u^{2}(t) \\
y(t)=1.5004 \cdot y(t-1)-0.7028 \cdot y(t-2) \\
+1.0538 \cdot v(t-1)-0.8463 \cdot v(t-2)+e(t)
\end{array}\right.
$$

In noise condition, after simulation, choose the best result as follows:

BPSO algorithm

$$
\left\{\begin{array}{l}
v(t)=1.6058 \cdot u(t)+1.5055 \cdot u^{2}(t) \\
y(t)=1.0484 \cdot y(t-1)-1.6257 \cdot y(t-2) \\
+0.7082 \cdot v(t-1)-0.1840 \cdot v(t-2)+e(t)
\end{array}\right.
$$

WPSO algorithm

$$
\left\{\begin{array}{l}
v(t)=1.4337 \cdot u(t)+1.3927 \cdot u^{2}(t) \\
y(t)=1.9962 \cdot y(t-1)-0.2973 \cdot y(t-2) \\
+0.6967 \cdot v(t-1)-0.3512 \cdot v(t-2)+e(t)
\end{array}\right.
$$

TPSO-SQP algorithm

$$
\left\{\begin{array}{l}
v(t)=0.7513 \cdot u(t)+0.7298 \cdot u^{2}(t) \\
y(t)=1.4708 \cdot y(t-1)-0.8712 \cdot y(t-2) \\
+0.8712 \cdot v(t-1)-0.5749 \cdot v(t-2)+e(t)
\end{array}\right.
$$

In no noise circumstance identified Hammerstein model by BPSO, WPSO and TPSO-SQP algorithm respectively fitting output of the system shown in figure 5 . In this case, model parameters estimation identified by TPSO-SQP algorithm are more close to the real value, namely using TPSO-SQP algorithm the accuracy of identification is improved significantly. From figure 6 we could see, in noise condition model parameters estimation identified by TPSO-SQP algorithm are also achieved a good effect, this fully show that TPSO-SQP algorithm is effectiveness and anti-interference performance. 


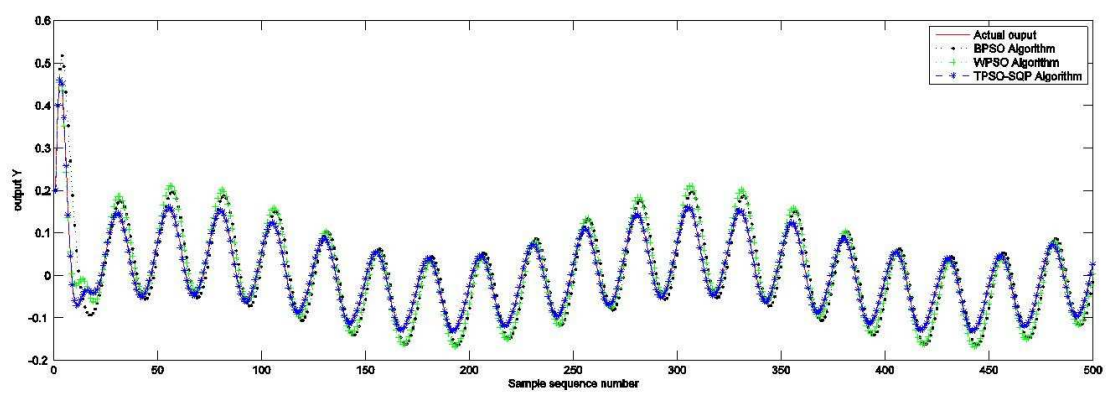

Fig. 5: System output curves of each algorithm in noiseless condition

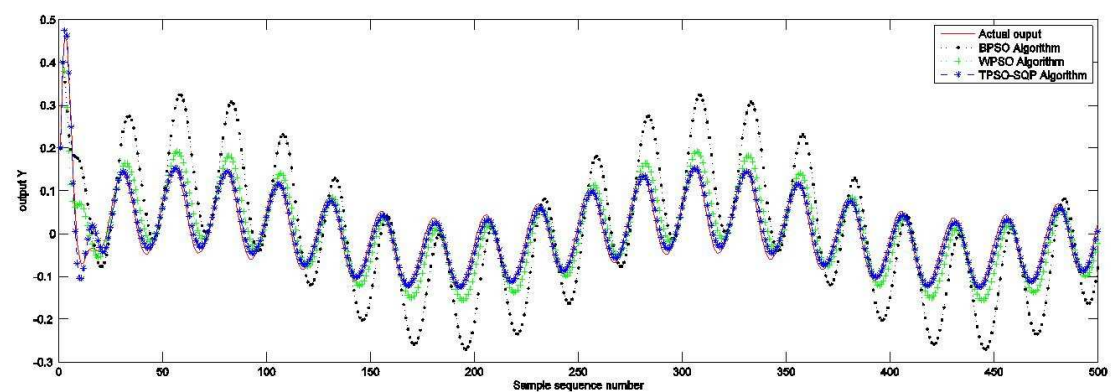

Fig. 6: System output curves of each algorithm in noise condition

Table 9: Identification parameters of each algorithm

\begin{tabular}{ccccccc}
\hline Truth Value & 0.3 & 0.6 & 1 & 1 & 0.3 & -0.4 \\
\hline BPSO & 0.3783 & 0.5251 & 0.8334 & 1.2468 & 0.2980 & -0.4104 \\
WPSO & 0.4377 & 0.4704 & 0.9265 & 0.9759 & 0.2950 & -0.3969 \\
TPSO-SQP & 0.3709 & 0.5229 & 1.0558 & 1.0280 & 0.2884 & -0.4130 \\
\hline
\end{tabular}

Table 10: Mean squares of each algorithm

\begin{tabular}{ccc}
\hline & maximum deviation & mean-square deviation \\
\hline BPSO & 0.2468 & 0.5570 \\
WPSO & 0.1377 & 0.4991 \\
TPSO-SQP & 0.0771 & 0.5440 \\
\hline
\end{tabular}

\subsection{Identification of Wiener Model}

Consider the following Wiener model

$$
\left\{\begin{array}{l}
x(t)=0.3 \cdot x(t-1)+0.6 \cdot x(t-2)+u(t)+\varepsilon(t) \\
y(t)=x^{3}(t)+0.3 \cdot x^{2}(t)-0.4 \cdot x(t)+e(t)
\end{array}\right.
$$

A Gaussian white noise is added to the output to test the robustness. We choose noise signal $e(t)$ which variance is 0.1 and mean value is 0 , pumping signal $u(t)$ is:

$$
u(t)=\frac{1}{20}\left[\sin \left(\frac{2 \pi}{250} t\right)+\sin \left(\frac{2 \pi}{25} t\right)\right]
$$

In noiseless condition, after simulation, choose the best result as follows:
BPSO algorithm

$$
\left\{\begin{array}{l}
x(t)=0.3783 \cdot x(t-1)+0.5251 \cdot x(t-2) \\
+0.8334 \cdot u(t)+\varepsilon(t) \\
y(t)=1.2468 \cdot x^{3}(t)+0.2980 \cdot x^{2}(t) \\
-0.4104 \cdot x(t)+e(t)
\end{array}\right.
$$

WPSO algorithm

$$
\left\{\begin{array}{l}
x(t)=0.4377 \cdot x(t-1)+0.4704 \cdot x(t-2) \\
+0.9265 \cdot u(t)+\varepsilon(t) \\
y(t)=0.9759 \cdot x^{3}(t)+0.2950 \cdot x^{2}(t) \\
-0.3969 \cdot x(t)+e(t)
\end{array}\right.
$$


Table 11: Identification parameters of each algorithm

\begin{tabular}{ccccccc}
\hline Truth Value & 0.3 & 0.6 & 1 & 1 & 0.3 & -0.4 \\
\hline BPSO & 0.0532 & 0.8324 & 1.3485 & 0.6730 & 0.2076 & -0.3320 \\
WPSO & 0.1709 & 0.7222 & 1.2113 & 0.7880 & 0.2348 & -0.3525 \\
TPSO-SQP & 0.2017 & 0.6557 & 1.3661 & 1.0967 & 0.2844 & -0.4305 \\
\hline
\end{tabular}

Table 12: Mean squares of each algorithm

\begin{tabular}{ccc}
\hline & maximum deviation & mean-square deviation \\
\hline BPSO & 0.4270 & 0.5994 \\
WPSO & 0.3120 & 0.5438 \\
TPSO-SQP & 0.3661 & 0.6522 \\
\hline
\end{tabular}

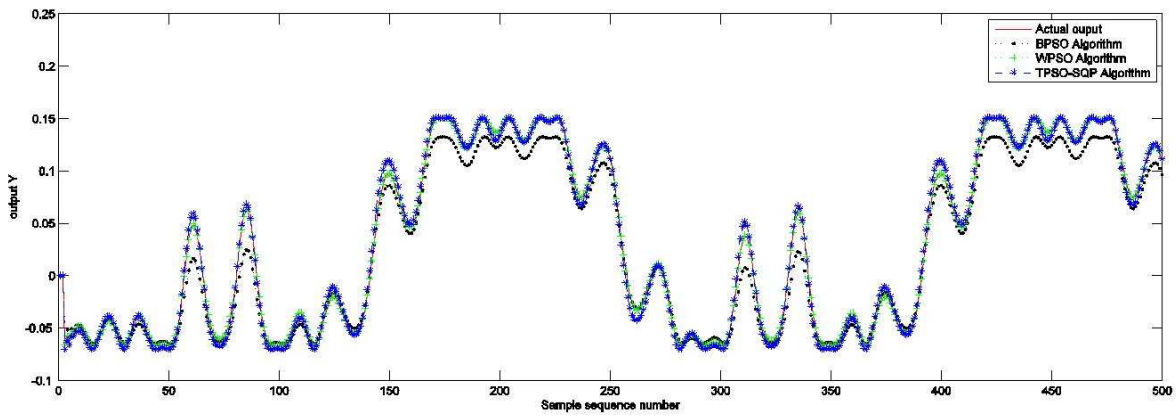

Fig. 7: System output curves of each algorithm in noiseless condition

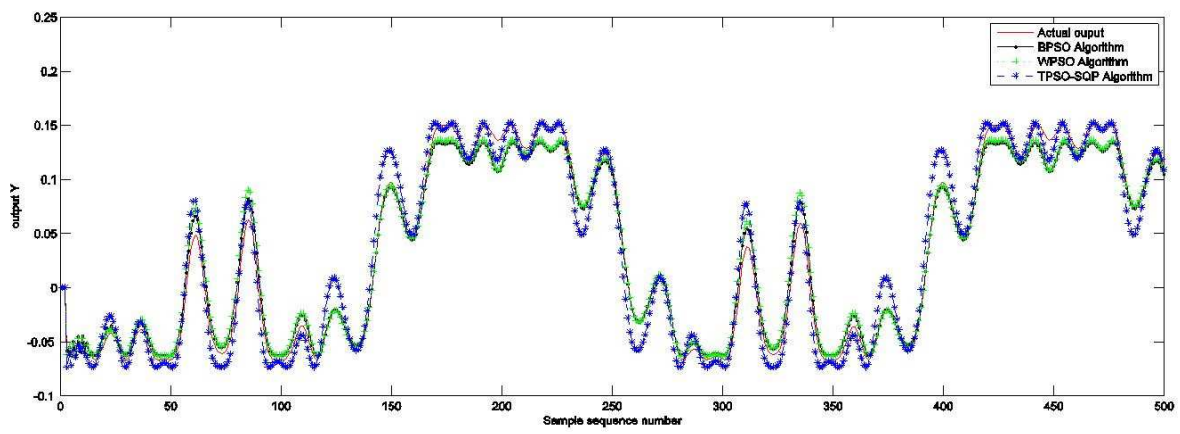

Fig. 8: System output curves of each algorithm in noise condition

TPSO-SQP algorithm

$$
\left\{\begin{array}{l}
x(t)=0.3709 \cdot x(t-1)+0.5299 \cdot x(t-2) \\
+1.0558 \cdot u(t)+\varepsilon(t) \\
y(t)=1.0280 \cdot x^{3}(t)+0.2884 \cdot x^{2}(t) \\
-0.4130 \cdot x(t)+e(t)
\end{array}\right.
$$

In noise condition, after simulation, choose the best result as follows:
BPSO algorithm

$$
\left\{\begin{array}{l}
x(t)=0.0532 \cdot x(t-1)+0.8324 \cdot x(t-2) \\
+1.3485 \cdot u(t)+\varepsilon(t) \\
y(t)=0.5730 \cdot x^{3}(t)+0.2076 \cdot x^{2}(t) \\
-0.3320 \cdot x(t)+e(t)
\end{array}\right.
$$

WPSO algorithm

$$
\left\{\begin{array}{l}
x(t)=0.1709 \cdot x(t-1)+0.7222 \cdot x(t-2) \\
+1.2113 \cdot u(t)+\varepsilon(t) \\
y(t)=0.6880 \cdot x^{3}(t-1)+0.2348 \cdot x^{2}(t) \\
-0.3525 \cdot x(t)+e(t)
\end{array}\right.
$$


TPSO-SQP algorithm

$$
\left\{\begin{array}{l}
x(t)=0.2017 \cdot x(t-1)+0.6557 \cdot x(t-2) \\
+1.3661 \cdot u(t)+\varepsilon(t) \\
y(t)=1.0967 \cdot x^{3}(t)+0.2844 \cdot x^{2}(t) \\
-0.4305 \cdot x(t)+e(t)
\end{array}\right.
$$

In no noise circumstance identified Wiener model by BPSO, WPSO and TPSO-SQP algorithm respectively fitting output of the system shown in figure 7. In this case, model parameters estimation identified by TPSO-SQP algorithm are more close to the real value, namely using TPSO-SQP algorithm the accuracy of identification is improved significantly. From figure 8 , we could see, in noise condition model parameters estimation identified by TPSO-SQP algorithm are also achieved a good effect, this fully shows that TPSO-SQP algorithm is effectiveness and anti-interference performance.

From the simulation results above, we can see that the noise factor has effect the algorithm identification performance, but TPSO-SQP algorithm can still identify parameters relative accuracy. In Hammerstein model and Wiener model identification system outputs really track real system output well. Therefore we could say that the TPSO-SQP algorithm is feasibility and effectiveness to nonlinear model identification.

\section{Conclusion}

In this paper we propose a method based on combination of a two-order oscillating with random inertia weight particle swarm optimization (TPSO) algorithm and successive quadratic programming (SQP) algorithm, namely TPSO-SQP. We show that the hybrid method has the advantage of both TPSO and SQP methods while does not inherent their drawbacks. The algorithm proposed by this paper point to the MIMO system presented a novel solution to system identification. The method converts system structure identification problem to best combination problem from typical models and all sub-models selection. TPSO-SQP combines the global search ability of TPSO algorithm with the local search ability of SQP algorithm. Avoid the defects of the two algorithm Simulation results show that the hybrid algorithm given by this paper is effective, high precision and well practicability.

\section{Acknowledgement}

This work is partially supported by the financial support of the National High-Tech Research and Development Plan of China (Grant 2008AA042131) and the National Grand Fundamental Research 973 Program of China (Grant 2007CB714300) and partly by the Automation Institute Beijing University of Chemical Technology. The authors are grateful to the anonymous reviewers for their valuable recommendations.

The authors are grateful to the anonymous referee for a careful checking of the details and for helpful comments that improved this paper.

\section{References}

[1] C. J. Fang, D. Y. Xiao, Process identification, Beijing: Tsinghua University Press, (1988).

[2] Y. J. Li, System identification theory and applications, Beijing: National Defense Industry Press, (2003).

[3] L. Wang, P. Ma, System identification methods, Electricity Information, 4, 63-66 (2001).

[4] X. Y. Li, Z. G. Han, Nonlinear system identification progress party, Automation Technology and Application, 23, 5-7 (2004).

[5] J. Kennedy, C. Eberhartr, Particle swarm optimization, International Conference on Neural Networks. Perth: IEEE, 1942-1948 (1995).

[6] F. Pan, J. Chen, M. G. Gan, et al, Model analysis of particle swarm optimization, Automation Technology, 32, 368-377 (2006).

[7] L. L. Zhu, Z. P. Yang, H. Yuan, Particle swarm optimization analysis and research, Computer Engineering and Applications, 43, 24-27 (2007).

[8] E. T. Omranm, A. Salman, Particle swarm optimization method for image clustering, International Journal of Pattern Recognition and Artificial Intelligence, 19, 297-321 (2005).

[9] T. Zhang, Multimode project scheduling based on particle swarm optimization, Computer Aided Civil and Infrastructure Engineering, 21, 93-103 (2006).

[10] S. A. Liu, F. Tang, System identification based on genetic algorithms method, Systems Engineering Theory and Practice, 16, 134-139 (2007).

[11] C. B. B. Costa, A. C. da Costa, R. Maciel Filho, Mathematical modeling and optimal control strategy development for an adipic acid crystallization process, Chemical Engineering and Processing, 44, 737-753 (2005).

[12] M. M. Zhu, Y. P. Wu, A PSO algorithm with high speed Convergence, Control and Decision, 25, 20-24 (2010).

[13] L. G. Wang, Q. H. Shi, Y. Hong, Hybrid Optimization Algorithm of PSO and AFSA, Computer Engineering, 36, 176-178 (2010).

[14] X. M. Liang, S. H. Dong, W. Long, PSO algorithm with dynamic inertia weight vector and dimension mutation, Journal of Computer Applications, 47, 29-31 (2011).

[15] Y. M. Liu, Q. Z. Zhao, B. Niu, Particle Swarm Optimization Algorithm Based on Dynamic Neighbor Topology Framework, Computer Engineering, 37, 210-212 (2011).

[16] Y. H. Sun, J. X. Wei, D. S. Xia, An Improved PSO Based on Diversity of Particle Symmetrical Distribution, Pattern Recognition and Artificial Intelligence, 23, 137-143 (2010).

[17] S. T. Li, M. K. Tan, I. W. Tsang, T. Y. K. James, A Hybrid PSO-BFGS Strategy for Global Optimization of Multimodal Functions, IEEE Transactions on Systems, Man, and Cybernetics, 41, 1003-1014 (2011). 
[18] J. Z. Zhang, X. M. Ding, A Multi-Swarm Self-Adaptive and Cooperative Particle Swarm Optimization, Engineering Applications of Artificial Intelligence, 24, 958-967 (2011).

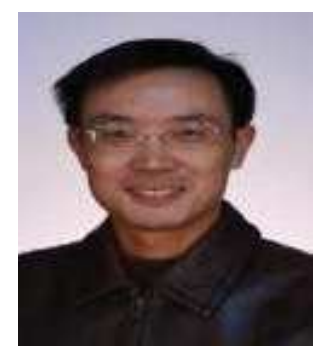

Qibing Jin received the MS degree from Northeast Petroleum University in 1996, and the $\mathrm{PhD}$ degree from the department of Optics Engineering, Northeastern University in 1999 . He is currently a professor in Beijing University of Chemical Technology. His research interests are in the areas of advanced control and application in industry, process modeling and optimization, and multivariate prediction and internal model control.

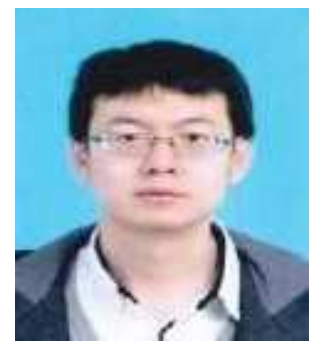

Kewen Wang received the MS degree from Liaoning Normal University in 2010, and the Phd degree from Institute of Automation in Beijing University of Chemical Technology in 2013. His research interests are in the areas of real-time database application, process modeling and optimization and system identification.

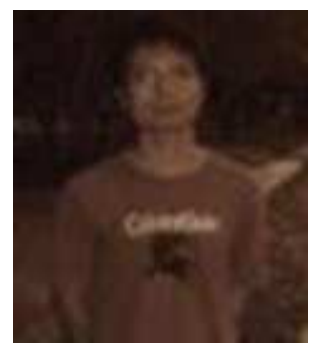

Jian Zhang received the MS degree from Institute of Automation in Beijing University of Chemical Technology in 2011. His research interests are in the areas of system identification theory and its application.

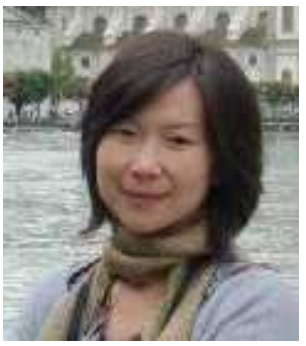

Liting Cao Lecturer at Beijing Union University and $\mathrm{Ph}$. D. candidate at the College of Information Science and Technology, Beijing University of Chemical Technology. She received her master degree from University of Science and Technology of China in 2005. Her research interest covers advanced control and its industrial application. 